\title{
Adiabatic Effective Action for Vortices in Neutral and Charged Superfluids
}

\author{
M. Hatsuda ${ }^{1}$, M. Sato ${ }^{2}$, S. Yahikozawa ${ }^{2}$ and T. Hatsuda ${ }^{3}$ \\ ${ }^{1}$ National Laboratory for High Energy Physics, Oho 1-1, Tsukuba, Ibaraki 305, Japan \\ 2 Physics Department, Kyoto University, Kyoto 606-01, Japan \\ ${ }^{3}$ Institute of Physics, University of Tsukuba, Tsukuba, Ibaraki 305, Japan
}

\begin{abstract}
Adiabatic effective action for vortices in neutral and charged superfluids at zero temperature are calculated using the topological Landau-Ginzburg theory recently proposed by Hatsuda, Yahikozawa, Ao and Thouless, and vortex dynamics are examined. The Berry phase term arising in the effective action naturally yields the Magnus force in both neutral and charged superfluids. It is shown that in neutral superfluid there is only one degree of freedom, namely the center of vorticities, and the vortex energy is proportinal to the sum of all vorticities so that it is finite only for the vanishing total vorticity of the system. On the other hand the effective mass and the vortex energy for a vortex in charged superfluids are defined individually as expected. The effects of the vortex core on these quantities are also estimated. The possible depinning scenario which is governed by the Magnus force and the inertial mass is also discussed.
\end{abstract}




\section{INTRODUCTION}

Vortex dynamics play an essential role in various properties of superfluids and superconductors [1,2]. Recently, numbers of experiments on the Hall effect 四 and the anomalous Hall effect [5] in superconductors have provided renewed interest in the theoretical study of vortex dynamics [6 14]. A standard theory of vortex dynamics has, however, not been established yet. For example, the existence of the Magnus force in superconductors is still an open question. In fact, Galilean invariance, which is used to derive the Magnus force in neutral superfluids, is unclear in charged superfluids because of the presence of a metallic lattice [2].

It is worth mentioning here that classical hydrodynamics [15] provides an explanation of two main properties of vortices in a perfect fluid; (i) Helmholtz's theorem and Kelvin's theorem lead to a vortex motion such that a vortex-line moves with the background fluid, and (ii) Bernoulli's theorem leads to the Magnus force acting on a vortex-line at rest. The nonlinear Schrödinger approach [3], which is one of the successful quantum descriptions for superconductors and superfluids, contains similar hydrodynamic equations: When the wave function is decomposed into the phase and the amplitude, $\psi=\sqrt{\rho} e^{\theta}$, the field equation for

$\theta$ becomes the continuity equation and the field equation for $\rho$ gives Bernoulli's theorem. Since this theory contains Bernoulli's theorem, it should lead to the Magnus force. In fact, there exists an attempt to calculate the expectation value of the Hall (transverse) conductivity and the longitudinal conductivity due to vortex motions by solving the timedependent nonlinear Schrödinger equation numerically [16]. However, it is a non-trivial task to extract the qualitative features of vortex dynamics, in particular the Magnus force, from such simulations.

In this paper, we develop an alternative approach to vortex dynamics, "the topological Landau-Ginzburg theory" which was proposed in a previous paper by two of us with Ao and Thouless [9]. This is a field theoretical approach whose field equations include the 
conventional nonlinear Schrödinger equation. In this approach, by using Helmholtz's and Kelvin's theorems as guiding principles, topological terms and collective coordinates for the vortex center are introduced. The hydrodynamical vortex law, " $\dot{\boldsymbol{X}}=\boldsymbol{v}(\boldsymbol{X})$ ", is naturally obtained by taking a variation of our "topological action". Furthermore, the vortex motion can be extracted even after taking into account the phonon and photon effects.

We will show that the topological term, " $\epsilon^{\mu \nu \rho \lambda} b_{\mu \nu} f_{\rho \lambda}$ ", and the source term in our action give rise to the Magnus force. This is consistent with the recent work by Ao and Thouless in which it is shown that the Berry phase and the dynamical phase in the quantum mechanical description of vortex system give rise to the Magnus force [6]. In our approach, the Magnus force appears naturally in both superconductors and superfluids, which is consistent with the expectation before [6] and with the recent results [10,11] in different approaches.

Our topological action also provides us with vortex-phonon couplings, where the phonon here is the density fluctuation of the order parameter at zero temperature, namely the zero sound wave. In actual superfluids and superconductors, there are situations where the vortices are trapped by pinning potentials instead of moving with the background flow. If the background flow becomes strong enough, depinning occurs and vortices start to move. A candidate of the driving force to overcome the pinning at zero temperature is the Magnus force [1,2], and the dynamics of depinning is dictated by the inertial mass of a vortex. An alternative candidate is quantum tunneling [17, but we do not consider it in this paper. We will calculate the inertial mass as well as the vortex energy in neutral and charged superfluids by taking into account the phonon and photon contributions. Also evaluated are the core contributions to the inertial mass and the vortex energy in charged superfluids where the size of a vortex-core is generally larger than the atomic scale. The obtained inertial mass is applicable to a wider range of the parameter region than that obtained in the phenomenological time-dependent Landau-Ginzburg theory supplemented with the Fermi liquid theory 18 .

The organization of this paper is as follows. In section II, a close analogy between the 
Lorentz force in electrodynamics and the Magnus force in hydrodynamics is discussed using our topological action for the superfluid without vortex-phonon interaction. In section III, the effective vortex dynamics in a superfluid are studied by taking into account the phonon interaction. In particular, the inertial mass and vortex energy are calculated. In section IV, vortex dynamics in a superconductor are examined by taking into account the photon interaction and the density fluctuations. The inertial mass and vortex energy in conventional and high $T_{c}$ superconductors are then evaluated. Section $\mathrm{V}$ is devoted to summary and concluding remarks.

\section{THE MAGNUS FORCE}

In this section, by using the topological Landau-Ginzburg theory without phonon/photon fluctuations, we will demonstrate that the origin of the Magnus force in superfluids is quite analogous to that of the Lorentz force in electrodynamics.

The action of topological Landau-Ginzburg theory for the neutral superfluid reads

$$
\begin{gathered}
S=\int d^{4} x\left[\psi^{*}\left(i \hbar \partial_{0}+\hbar a_{0}\right) \psi-\frac{1}{2 m}\left|\left(i \hbar \partial_{i}+\hbar a_{i}\right) \psi\right|^{2}-g\left(|\psi|^{2}-\rho_{0}\right)^{2}\right. \\
\left.+\frac{\hbar}{2 m} \varepsilon^{\mu \nu \rho \sigma} b_{\mu \nu} f_{\rho \sigma}+b_{\mu \nu} J^{\mu \nu}\right]-U_{p i n}(X) \\
J^{\mu \nu}(x)=\sum_{a=1}^{N} \gamma_{a} \int d \tau d \sigma \frac{\partial X_{a}^{[\mu}}{\partial \tau} \frac{\partial X_{a}^{\nu]}}{\partial \sigma} \delta^{(4)}\left(x-X_{a}(\sigma, \tau)\right)
\end{gathered}
$$

where $\gamma_{a}=2 \pi n_{a} \hbar / m$ with integer $n_{a}$ and $2 \pi \hbar / m$ denotes vorticity unit. Notations follow from our previous paper [9]. $a_{\mu}$ is a vector potential representing vortex singularities, $b_{\mu \nu}$ is a rank-two anti-symmetric tensor potential, $J^{\mu \nu}$ is the vorticity current, and $U_{\text {pin }}(X)$ is a

pinning potential. We examine vortex dynamics by taking a variation of $S$ with respect to the vortex coordinate $X$.

There is a one-to-one correspondence of our theory to electrodynamics where a point-like charged particle has a current 


$$
J^{\mu}(x)=e \int d \tau \frac{d X^{\mu}}{d \tau} \delta^{(4)}(x-X(\tau))
$$

Variation of the source term $-\int d^{4} x A_{\mu} J^{\mu}$ in electrodynamics with respect to the particle coordinate $X(t)$ gives the Lorentz force

$$
\begin{aligned}
-\frac{\delta}{\delta X^{i}(t)} \int d^{4} x A_{\mu} J^{\mu} & =-e F_{i \mu}(X) \frac{d X^{\mu}(t)}{d t} \\
& =e(\boldsymbol{E}(X)+\dot{\boldsymbol{X}} \times \boldsymbol{B}(X))^{i} \\
& =F_{\text {Lorentz }}^{i}(X)
\end{aligned}
$$

where the proper-time variable $\tau$ is chosen to be $\tau=t=X^{0}$.

Analogously a variation of the source term $\int d^{4} x b J$ in (11) with respect to the vortex coordinate gives

$$
\frac{\delta}{\delta X^{i}(t, \sigma)} \int d^{4} x b_{\mu \nu} J^{\mu \nu}=\gamma H_{i \mu \nu}(X) \frac{\partial X^{[\mu}}{\partial t} \frac{\partial X^{\nu]}}{\partial \sigma}
$$

where $H_{\mu \nu \rho}=\partial_{\mu} b_{\nu \rho}+\partial_{\nu} b_{\rho \mu}+\partial_{\rho} b_{\mu \nu}$ and $\tau=t=X^{0}$. On the other hand, the field equation for $a_{\mu}$ obtained from (1) gives a relation between the $b$-field and the hydrodynamical current, $\mathcal{J}^{\mu}(x)=\left(m \rho(x), m \rho(x) v^{i}(x)\right)$;

$$
H_{\mu \nu \rho}=-\frac{1}{2} \varepsilon_{\mu \nu \rho \lambda} \mathcal{J}^{\lambda}
$$

The $b$-field is the antisymmetric rank-two tensor potential whose exterior derivative is the observable hydrodynamical current. This property is analogous to the vector potential whose exterior derivative is electromagnetic field. Note that $H_{i \mu \nu}(X)$ in eq.(5) should be considered as an external field acting on the vortex at point $X$, which is analogous to the electromagnetic field acting on the charged particle in eq.(41). Inserting eq.(6) into eq.(5) leads to the conventional Magnus force

$$
\begin{aligned}
\frac{\delta}{\delta X^{i}(t, \sigma)} \int b_{\mu \nu} J^{\mu \nu} d x & =-\varepsilon_{i \mu \nu \rho} \mathcal{J}^{\rho}(X) \frac{\partial X^{\mu}}{\partial t}\left(\gamma \frac{\partial X^{\nu}}{\partial \sigma}\right) \\
& =-m \rho(X)[(\dot{X}-\boldsymbol{v}(X)) \times \boldsymbol{\omega}(X)]^{i} \\
& =F_{\text {Magnus }}^{i}(X)
\end{aligned}
$$


where we have used the fact that $\left(\gamma \frac{\partial \boldsymbol{X}}{\partial \sigma}\right)$ is the vorticity vector, $\boldsymbol{\omega} \equiv \nabla \times \boldsymbol{v}$. The Magnus force, which is analogous to the Lorentz force in electrodynamics, is thus the fundamental force in the hydrodynamic theory.

If there are no pinning potentials, the variation of $S$ at the "classical" level $\partial S / \partial X^{i}(t, \sigma)=0$ gives an equation of a vortex $F_{\text {Magnus }}^{i}(X)=-m \rho(X)[(\dot{\boldsymbol{X}}-\boldsymbol{v}(X)) \times$ $\boldsymbol{\omega}(X)]^{i}=0$. Analogous to the case of the electrodynamics, $\mathcal{J}^{\mu}(X)$ and $H^{\mu \nu \rho}(X)$ take values at point $X$ without the contribution of a vortex sitting at $X$ in the weak current limit; namely $\rho(X) \neq 0$ and $\boldsymbol{v}(X) \neq 0$. Thus one arrives at the conventional classical hydrodynamical relation $\dot{\boldsymbol{X}}=\boldsymbol{v}(\boldsymbol{X})$ which means that the vortex moves with the same velocity as the background flow. Even if one takes into account the full vortex contributions to $\mathcal{J}^{\mu}(X)$, as far as one considers the finite size of a vortex core, it is possible to derive similar hydrodynamical relation in an averaged sense:

$$
\langle\dot{\boldsymbol{X}}\rangle=\frac{\langle\rho \boldsymbol{v}(\boldsymbol{X})\rangle}{\langle\rho(\boldsymbol{X})\rangle}
$$

In fact, if one uses a distribution with finite core size instead of the $\delta$-function distribution given in (2), the vortex centers $X_{a}$ and the current $\mathcal{J}^{\mu}(X)$ must be defined by averaging over the distribution as denoted in by $\langle\cdot\rangle$ in (8). For "classical hydrodynamical" cases (8) further reduces to $\langle\dot{X}\rangle=\langle\boldsymbol{v}(\boldsymbol{X})\rangle$ since $\langle\rho \boldsymbol{v}(\boldsymbol{X})\rangle=\langle\rho(\boldsymbol{X})\rangle\langle\boldsymbol{v}(\boldsymbol{X})\rangle$ holds. One can imagine easily this situation for a case such as an isolated straight vortex line along the $z$-direction and a constant background flows in the $\mathrm{x}-\mathrm{y}$ plane.

The above discussions are valid not only for neutral superfluids but also for charged superfluids since the $b J$ coupling is universal in both cases. Namely the Magnus force is a fundamental force in both neutral and charged superfluids.

Up to this point, we have not taken into account the interactions of phonons or photons with vortices. They actually induce a kinietic term $\frac{1}{2} m_{\text {eff }} \dot{X}^{2}$ with $m_{\text {eff }}$ being the inertial mass. This means that, beyond the "classical" level of $S$, the acceleration term is induced from the equation of motion of $X$, i.e., $m_{\text {eff }} \dot{X}^{2}=F_{\text {Magnus }}(X)+F_{\text {pin }}(X)$. This will be 
discussed in detail in later sections.

\section{EFFECTIVE ACTION FOR VORTICES IN NEUTRAL SUPERFLUID}

Now let us calculate the effective action for vortices in a neutral superfluid by taking into account the phonon interaction at zero temperature. We begin with the topological Landau-

Ginzburg theory (1) and (2) and define the phase and amplitude variables as $\psi=\sqrt{\rho(x)} e^{i \theta(x)}$. Thus the action $S$ reads

$$
\begin{aligned}
S=\int d^{4} x\left[\hbar \rho\left(-\partial_{0} \theta+a_{0}\right)\right. & -\frac{\hbar^{2} \rho}{2 m}(\nabla \theta+\boldsymbol{a})^{2}-\frac{\hbar^{2}}{8 m \rho}(\nabla \rho)^{2}-g\left(\rho-\rho_{0}\right)^{2} \\
& \left.+\frac{\hbar}{2 m} \varepsilon^{\mu \nu \rho \sigma} b_{\mu \nu} f_{\rho \sigma}+b_{\mu \nu} J^{\mu \nu}\right]-U_{p i n}(X)
\end{aligned}
$$

If there is a uniform background flow $\boldsymbol{v}_{b g}=$ constant and vortex excitations do not exist, the stationary state of the superfluid is characterized as

$$
a^{\mu}=0 \quad, \quad \rho=\rho_{0} \quad, \quad \theta=\theta_{b g} \equiv \frac{m}{\hbar} \boldsymbol{v}_{b g} \cdot \boldsymbol{r}-\frac{m}{2 \hbar} \boldsymbol{v}_{b g}^{2} t \quad .
$$

In order to take into account the phonon fluctuation, we expand the amplitude and phase variables around the above stationary solution, $\rho(x)=\rho_{0}+\delta \rho, \theta(x)=\theta_{b g}+\delta \theta$. Then the full functional integral and the effective action $S_{\text {eff }}(X)$ can be written as

$$
\mathcal{Z}=\int \mathcal{D}\left[\delta \rho, \delta \theta, a_{\mu}, b_{\mu \nu}\right] e^{i S\left(\rho, \theta, a_{\mu}, b_{\mu \nu} ; X\right)}=e^{i S_{e f f}(X)}
$$

where $S\left(\rho, \theta, a_{\mu}, b_{\mu \nu} ; X\right)=\int d^{4} x \mathcal{L}-U_{\text {pin }}(X)$ with

$$
\begin{aligned}
& \mathcal{L}=\mathcal{L}_{s t}+ \delta \rho\left(\hbar a_{0}-\hbar \boldsymbol{v}_{b g} \cdot \boldsymbol{a}-\frac{\hbar^{2}}{2 m} \boldsymbol{a}^{2}\right)+\frac{1}{2}\left(\begin{array}{ll}
\delta \rho & \delta \theta
\end{array}\right) G^{-1}\left(\begin{array}{c}
\delta \rho \\
\delta \theta
\end{array}\right) \\
&-\frac{\hbar^{2}}{m} \delta \rho \boldsymbol{a} \cdot \nabla \delta \theta+O\left(\delta^{3}\right)+(b f, \quad b J), \\
& \mathcal{L}_{s t}=\rho_{0}\left(\hbar a_{0}-\hbar \boldsymbol{v}_{b g} \cdot \boldsymbol{a}-\frac{\hbar^{2}}{2 m} \boldsymbol{a}^{2}\right) .
\end{aligned}
$$


Here $\mathcal{L}_{s t}$ is a stationary part of the action and $G^{-1}$ is a Hermitian matrix defined as

$$
G^{-1}=\left(\begin{array}{cc}
\left(-2 g+\frac{\hbar^{2}}{4 m \rho_{0}} \triangle\right) & \left(-\hbar \partial_{0}-\hbar \boldsymbol{v}_{b g} \cdot \nabla\right) \\
\left(\hbar \partial_{0}+\hbar \boldsymbol{v}_{b g} \cdot \nabla\right) & \frac{\rho_{0} \hbar^{2}}{m} \triangle
\end{array}\right)
$$

where we used the Coulomb gauge for the $a$-field and divergence free property of the background flow. $G$ is nothing but the Green's function for the fluctuations and has poles at

$$
\omega=\boldsymbol{v}_{b g} \cdot \boldsymbol{k} \pm \epsilon(\boldsymbol{k}), \quad \text { with } \quad \epsilon^{2}(\boldsymbol{k})=c_{s}^{2} \boldsymbol{k}^{2}+\left(\frac{\boldsymbol{k}^{2}}{2 m}\right)^{2}
$$

with $\epsilon(\boldsymbol{k})$ being the Bogoliubov spectrum and $c_{s}$ being the zero sound velocity

$$
c_{s}=\sqrt{\frac{2 \rho_{0} g}{m}} \text {. }
$$

For small momentum $\boldsymbol{k}$, this reduces to the massless phonon mode propagating with sound velocity under the background flow. After the Gaussian integration with respect to $\delta \rho$ and $\delta \theta$, the effective lagrangian becomes

$$
\begin{aligned}
& \mathcal{L}_{e f f}\left(a_{\mu}, b_{\mu \nu} ; X\right) \\
& \approx \mathcal{L}_{s t}+\frac{\rho_{0} \hbar^{2}}{2 m}\left(a_{0}-\boldsymbol{v}_{b g} \cdot \boldsymbol{a}-\frac{\hbar}{2 m} \boldsymbol{a}^{2}\right)\left(\frac{-\triangle}{\left(\partial_{0}+\boldsymbol{v}_{b g} \cdot \nabla\right)^{2}+\epsilon^{2}}\right)\left(a_{0}-\boldsymbol{v}_{b g} \cdot \boldsymbol{a}-\frac{\hbar}{2 m} \boldsymbol{a}^{2}\right) \\
&+\frac{\hbar}{2 m} \varepsilon^{\mu \nu \rho \sigma} b_{\mu \nu} f_{\rho \sigma}+b_{\mu \nu} J^{\mu \nu} \\
& \approx \mathcal{L}_{s t}+\frac{\rho_{0} \hbar^{2}}{2 m} \frac{1}{c_{s}^{2}}\left(a_{0}-\boldsymbol{v}_{b g} \cdot \boldsymbol{a}-\frac{\hbar}{2 m} \boldsymbol{a}^{2}\right)^{2}+\frac{\hbar}{2 m} \varepsilon^{\mu \nu \rho \sigma} b_{\mu \nu} f_{\rho \sigma}+b_{\mu \nu} J^{\mu \nu}
\end{aligned}
$$

For interactions between vortices, only the phonon modes with small momentum are important under normal circumstances, since the vortex motion and the background flow are much slower than the zero sound velocity; $\dot{X}<<c_{s}, v_{b g}<<c_{s}$. Therefore, from the second equality to the last one in (16), $\left(\partial_{0}+\boldsymbol{v}_{b g} \cdot \nabla\right)^{2}$ is neglected and $\epsilon^{2} \approx-c_{s}^{2} \triangle$ is taken (the adiabatic approximation). 
The integration of the $b_{\mu \nu}$ field is straightforward and the following constraint on $a_{\mu}$ is obtained:

$$
f_{\rho \lambda}=\partial_{\rho} a_{\lambda}-\partial_{\lambda} a_{\rho}=\frac{m}{2 \hbar} \varepsilon_{\rho \lambda \mu \nu} J^{\mu \nu} \quad .
$$

This constraint (17) can be solved in the Coulomb gauge as

$$
\left\{\begin{array}{l}
a_{0}=\frac{-1}{\triangle}(m / 2 \hbar) \epsilon_{i j k} \partial^{i} J^{j k} \\
a_{i}=\frac{-1}{\triangle}(m / \hbar) \epsilon_{i j k} \partial^{j} J^{0 k}
\end{array} .\right.
$$

In the following, we will mostly focus our attention on the vortices in two spatial dimensions where vortices lie along the z-direction to make the argument as simple as possible. In this case, the $a_{\mu}$ field is written as

$$
\left\{\begin{array}{l}
a_{0}=-\sum_{a} n_{a} \boldsymbol{e}_{z} \cdot\left(\dot{\boldsymbol{X}}_{a} \times \nabla \ln \left|\boldsymbol{x}-\boldsymbol{X}_{a}\right|\right) \\
\boldsymbol{a}=\sum_{a} n_{a} \boldsymbol{e}_{z} \times \nabla \ln \left|\boldsymbol{x}-\boldsymbol{X}_{a}\right|
\end{array} .\right.
$$

We will now insert the solutions (19) into (16) to get an effective action $S_{\text {eff }}(X)$ written in the vortex coordinate alone. When doing this, it is convenient to classify terms by the number of $a_{\mu}$-fields. The $a_{\mu}$ field contains the vortex singularities, so the number of $a_{\mu}$ fields represents the number of the interacting vortices; for example, a bilinear term of $a_{\mu}$ field contains an interaction of two vortices. Let us first evaluate the term linear in $a_{\mu}$ field. Inserting the expressions (19) into the linear term in (16), one gets

$$
\begin{aligned}
\int d^{2} x \rho_{0} \hbar\left(a_{0}-\boldsymbol{v}_{b g} \cdot \boldsymbol{a}\right) & =-\int d^{2} x \rho_{0} \hbar \sum_{a} n_{a} \boldsymbol{e}_{z} \cdot\left(\dot{\boldsymbol{X}}_{a}-\boldsymbol{v}_{b g}\right) \times \nabla \ln \left|\boldsymbol{x}-\boldsymbol{X}_{a}\right| \\
& =\frac{m \rho_{0}}{2} \sum_{a} \gamma_{a} \boldsymbol{e}_{z} \cdot\left(\dot{\boldsymbol{X}}_{a}-\boldsymbol{v}_{b g}\right) \times\left(\boldsymbol{X}_{a}-\boldsymbol{v}_{b g} t\right)
\end{aligned}
$$

where one should be careful to perform spatial integration under the background flow. In fact, there is a constant ambiguity in the integral, $\int d^{2} x \nabla \ln |\boldsymbol{x}-\boldsymbol{X}|=-\pi(\boldsymbol{X}+\boldsymbol{c}) . \quad \boldsymbol{c}$ can be fixed as $-\boldsymbol{v}_{b g} t$ by requiring a correct boundary condition, i.e., the boundary of the 
superfluid should also move with the background flow velocity. From (20), one sees that the combination $a_{0}-\boldsymbol{v}_{b g} \cdot \boldsymbol{a}$ has the Galilean invariance.

The obtained expression (20) is a generalization of the Berry phase term [20] to the system with background flow. It is easy to show that taking a variation of this term with respect to the vortex coordinates leads to the Magnus force.

Secondly, let us evaluate bilinear terms in the $a_{\mu}$ field. In order to evaluate them we will use the following formula:

$$
\begin{aligned}
& \int d^{2} x \partial_{i} \ln \left|\boldsymbol{x}-\boldsymbol{X}_{a}\right| \partial_{j} \ln \left|\boldsymbol{x}-\boldsymbol{X}_{b}\right| \\
& \quad= \begin{cases}-\pi\left\{\delta^{i j} \ln \left(\left|\boldsymbol{X}_{a b}\right| / R\right)+\left(X_{a b}^{i} X_{a b}^{j} / \boldsymbol{X}_{a b}^{2}-\delta^{i j} / 2\right)\right\} & \text { for } \boldsymbol{X}_{a} \neq \boldsymbol{X}_{b} \\
-\pi \delta^{i j} \ln (d / R) & , \text { for } \boldsymbol{X}_{a}=\boldsymbol{X}_{b}\end{cases}
\end{aligned}
$$

where $d$ is an ultraviolet cutoff which is an atomic scale, $R$ is an infrared cutoff and $\boldsymbol{X}_{a b} \equiv$ $\boldsymbol{X}_{a}-\boldsymbol{X}_{b}$. Appendix A contains a derivation of the above formula by regulating $\ln |\boldsymbol{x}|$ by $\ln \sqrt{\boldsymbol{x}^{2}+\varepsilon^{2}}$ with $d=\varepsilon e^{1 / 2}$. It is natural to choose $R$ to be the container size. In a charged superfluid, the penetration depth plays the role of the infrared cutoff $R$, since the charge screening makes the interaction region finite.

Using the formula (21), $\int \boldsymbol{a}^{2}$ term in (12) and (16) becomes

$$
\begin{aligned}
-\frac{\rho_{0} \hbar^{2}}{2 m} \int d^{2} x \boldsymbol{a}^{2} & =-\frac{\rho_{0} \hbar^{2}}{2 m}\left(\sum_{a=b}+\sum_{a \neq b}\right) n_{a} n_{b} \int d^{2} x \nabla \ln \left|\boldsymbol{x}-\boldsymbol{X}_{a}\right| \cdot \nabla \ln \left|\boldsymbol{x}-\boldsymbol{X}_{b}\right| \\
& =-\frac{\rho_{0} \hbar^{2} \pi}{m}\left\{\sum_{a} n_{a}^{2} \ln \frac{R}{d}+\sum_{a \neq b} n_{a} n_{b}\left(\ln \frac{R}{d}-\ln \frac{\left|\boldsymbol{X}_{a b}\right|}{d}\right)\right\} \\
& =-E_{0}+\sum_{a \neq b} \frac{m \rho_{0}}{4 \pi} \gamma_{a} \gamma_{b} \ln \frac{\left|\boldsymbol{X}_{a b}\right|}{d}
\end{aligned}
$$

Here $E_{0}$ is the static energy for a system with many vortices,

$$
E_{0}=\frac{m \rho_{0}}{4 \pi}\left(\sum_{a} \gamma_{a}\right)^{2} \ln \frac{R}{d}
$$

The obtained expression $E_{0}$ for an isolated vortex coincides with Feynman's result [21]. If the container size is infinite, $E_{0}$ for a single vortex is divergent, while $E_{0}$ vanishes for a 
system with zero total vorticity. For example, a vortex - anti-vortex pair has zero static energy and has only the logarithmic interaction in (22).

Among the self-interaction terms, there is a term which is interpreted as the kinetic term;

$$
\begin{array}{rl}
\int d^{2} & x \frac{\rho_{0} \hbar^{2}}{2 m c_{s}^{2}}\left(a_{0}-\boldsymbol{v}_{b g} \cdot \boldsymbol{a}\right)^{2} \\
& =\frac{\rho_{0} \hbar^{2}}{2 m c_{s}^{2}}\left(\sum_{a=b}+\sum_{a \neq b}\right) n_{a} n_{b}\left(\dot{\boldsymbol{X}}_{a}-\boldsymbol{v}_{b g}\right)^{i}\left(\dot{\boldsymbol{X}}_{b}-\boldsymbol{v}_{b g}\right)^{j} \epsilon^{i k} \epsilon^{j l} \int d^{2} x \partial_{k} \ln \left|\boldsymbol{x}-\boldsymbol{X}_{a}\right| \partial_{l} \ln \left|\boldsymbol{x}-\boldsymbol{X}_{b}\right| \\
& =\frac{1}{2} m_{e f f}\left(\sum_{a} \frac{\gamma_{a}}{\gamma_{0}}\left(\dot{\boldsymbol{X}}_{a}-\boldsymbol{v}_{b g}\right)\right)^{2} \\
& -\frac{m \rho_{0}}{8 \pi c_{s}^{2}} \sum_{a \neq b} \gamma_{a} \gamma_{b}\left\{\left(\dot{\boldsymbol{X}}_{a}-\boldsymbol{v}_{b g}\right) \cdot\left(\dot{\boldsymbol{X}}_{b}-\boldsymbol{v}_{b g}\right)\left(\ln \frac{\left|\boldsymbol{X}_{a b}\right|}{d}+\frac{1}{2}\right)-\left(\dot{\boldsymbol{X}}_{a}-\boldsymbol{v}_{b g}\right) \cdot \boldsymbol{u}_{a b}\left(\dot{\boldsymbol{X}}_{b}-\boldsymbol{v}_{b g}\right) \cdot \boldsymbol{u}_{a b}\right\}
\end{array}
$$

where $\boldsymbol{u}_{a b} \equiv \boldsymbol{X}_{a b} /\left|\boldsymbol{X}_{a b}\right|$. Here we define

$$
\boldsymbol{X}_{C V} \equiv \sum_{a} \frac{\gamma_{a}}{\gamma_{0}}\left(\boldsymbol{X}_{a}-\boldsymbol{v}_{b g} t\right) \quad, \quad \gamma_{0} \equiv \frac{2 \pi \hbar}{m}
$$

and call this "the center of vorticity" of the system. This is analogous to the center of mass except that vorticity takes both positive and negative value. Then the first term of eq.(24) is interpreted as a kinetic term for $\boldsymbol{X}_{C V}$ with the following inertial mass

$$
m_{e f f}=\frac{m \rho_{0}}{4 \pi c_{s}^{2}} \gamma_{0}^{2} \ln \frac{R}{d}
$$

If phonon fluctuations are neglected (i.e., in the limit $c_{s} \rightarrow 0$ ), "the center of vorticity" $\sum \gamma_{a} \boldsymbol{X}_{a} / \gamma_{0}$ is conserved since it is proportional to the conserved total momentum $\boldsymbol{P}=$ $\sum \boldsymbol{p}_{a}=-\left(m \rho_{0} / 2\right) \boldsymbol{e}_{z} \times \sum \gamma_{a} \boldsymbol{X}_{a}$. If there exist phonons, $\boldsymbol{P}$ has an extra term from the phonon momentum and $\sum \gamma_{a} \boldsymbol{X}_{a}$ is not conserved anymore. Instead, it gets mobility with an inertial mass $m_{\text {eff }}$ given in (26).

The magnitude of the vortex energy $E_{0}$ in (23) and the inertial mass $m_{\text {eff }}$ in (26), are consistent with those in ref. [18] and ref. [19] obtained by different approaches, although the vorticity dependence was not considered in these references. Ref. [18] is based on a 
phenomenological time-dependent Landau-Ginzburg theory supplemented with the Fermi liquid theory, while ref. [19] is based on the functional integral approach at finite temperature. It is, however, unclear whether our result is consistent with ref. [8] which discusses the behavior of $m_{e f f}$ for large $R$.

Lastly we evaluate the vortex interactions. The leading term of two-vortex interaction is given by the second term of the right hand side of (22). Next to leading terms such as the second term in (24) are suppressed by the factor $\boldsymbol{v}_{b g}^{2} / c_{s}^{2}$ which is of order $10^{-4} \sim 10^{-8}$ for superfluids $\mathrm{He}^{4}, 10^{-2}$ for conventional superconductors and $10^{-6}$ for high $T_{c}$ superconductors. The suppression factor $\dot{\boldsymbol{X}}^{2} / c_{s}^{2}$ is even smaller than $\boldsymbol{v}_{b g}^{2} / c_{s}^{2}$ because we consider the adiabatic motion of vortices. Three and four vortex interactions are generated from third order and fourth order terms in $a_{\mu}$. However, they can be neglected in a dilute vortex system. Furthermore these terms are suppressed by $1 / c_{s}^{2}$ and $\hbar$. Third and fourth order terms also contain contributions to the vortex self-energy and the two-vortex interactions, but they are suppressed by $\hbar$ compared to leading terms in (22) which are already of $O\left(\hbar^{2}\right)$.

The resultant effective action up to $O\left(\hbar^{2}\right)$ and $O\left(\left(1 / c_{s}^{0}\right)\right)$ is summarized as

$$
\begin{gathered}
S_{\text {eff }}(X)=\int d t\left[L_{\text {Berry }}+L_{v e}+L_{k i n}+L_{i n t}\right]-U_{\text {pin }}(X), \\
\left\{\begin{array}{l}
L_{\text {Berry }}=\frac{m \rho_{0}}{2} \sum_{a} \gamma_{a} \boldsymbol{e}_{z} \cdot\left(\dot{\boldsymbol{X}}_{a}-\boldsymbol{v}_{b g}\right) \times\left(\boldsymbol{X}_{a}-\boldsymbol{v}_{b g} t\right) \\
L_{v e}=-E_{0}, \quad \text { with } \quad E_{0}=\left(m \rho_{0} / 4 \pi\right)\left(\sum_{a} \gamma_{a}\right)^{2} \ln (R / d) \\
L_{k i n}=\frac{1}{2} m_{e f f} \dot{\boldsymbol{X}}_{C V}^{2}, \quad \text { with } \quad m_{e f f}=\left(m \rho_{0} / 4 \pi c_{s}^{2}\right) \gamma_{0}^{2} \ln (R / d) \\
L_{i n t}=\sum_{a \neq b}\left(m \rho_{0} / 4 \pi\right) \gamma_{a} \gamma_{b} \ln \left(\left|\boldsymbol{X}_{a b}\right| / d\right),
\end{array}\right.
\end{gathered}
$$

where $L_{\text {Berry }}, L_{v e}, L_{k i n}$ and $L_{\text {int }}$ are the Berry phase term, the vortex energy, the vortex kinetic term and the interaction term, respectively. 
The equation of motion for an isolated vortex with $n_{a}=1$ at zero temperature is determined by varying the effective action which is the sum of the acceleration term, the Magnus force, and the pinning force;

$$
\begin{aligned}
m_{e f f} \ddot{\boldsymbol{X}} & =-m \rho_{0}\left(\dot{\boldsymbol{X}}-\boldsymbol{v}_{b g}\right) \times \boldsymbol{\omega}+\boldsymbol{F}_{\text {pin }} \\
& =\boldsymbol{F}_{\text {Magnus }}+\boldsymbol{F}_{\text {pin }} .
\end{aligned}
$$

This equation gives the following scenario for vortex pinning and depinning. Consider a vortex pinned by the pinning potential $U_{\text {pin }}(X)$. The vortex feels the Magnus force as long as it does not move with the background flow $\boldsymbol{v}_{b g}$. If the Magnus force is strong enough to overcome the pinning potential, the vortex starts to move (depinning) with the accerelation dictated by the inertial mass $m_{\text {eff }}$. As the vortex velocity gets closer to the background velocity $\boldsymbol{v}_{b g}$, the Magnus force gets smaller and reduces eventually to zero. The

final stationary situation is then described by the hydrodynamical law for a perfect fluid; $\dot{X}=v(X)$. The phonon interaction is irrelevant at the final stage, but it is essential at the depinning stage.

\section{EFFECTIVE ACTION FOR VORTICES IN CHARGED SUPERFLUID}

Now let us consider vortex dynamics in type II superconductors. We propose the following topological Landau-Ginzburg theory for vortices in superconductors;

$$
\begin{gathered}
S=\int d^{4} x\left[\psi^{*}\left(i \hbar \partial_{0}+\hbar a_{0}-\frac{q}{c} A_{0}\right) \psi-\frac{1}{2 m}\left|\left(i \hbar \partial_{i}+\hbar a_{i}-\frac{q}{c} A_{i}\right) \psi\right|^{2}-g\left(|\psi|^{2}-\rho_{0}\right)^{2}\right. \\
\left.-\rho_{l a t} A_{0}-\frac{1}{4} F^{2}+\frac{\hbar}{2 m} \varepsilon^{\mu \nu \rho \sigma} b_{\mu \nu} f_{\rho \sigma}+b_{\mu \nu} J^{\mu \nu}\right]-U_{p i n}(X) \\
J^{\mu \nu}(x)=\sum_{a=1}^{N} \gamma_{a} \int d \tau d \sigma \frac{\partial X_{a}^{[\mu}}{\partial \tau} \frac{\partial X_{a}^{\nu]}}{\partial \sigma} \delta^{(4)}\left(x-X_{a}(\sigma, \tau)\right)
\end{gathered}
$$

where $q=2 e$ and $m=2 m_{e}$ with $e$ and $m_{e}$ being the electron's charge and mass, $A_{\mu}$ being the electromagnetic potential and $F_{\mu \nu}=\partial_{\mu} A_{\nu}-\partial_{\nu} A_{\mu} \cdot \rho_{\text {lat }}$ is the electric charge of the 
background lattice. In the stationary situation without vortices, the total electric charge vanishes locally; $\rho_{\text {lat }}+(q / c) \rho_{0}=0$.

The Meissner effect and the quantization of magnetic flux can be easily checked by the variation of the action (29)). The variation with respect to $A^{i}$ gives

$$
\partial^{\mu} F_{\mu i}+J_{e}^{i}=0 \quad, \quad \boldsymbol{J}_{e}=-\frac{\rho q}{m c}\left(-\hbar \nabla \theta-\hbar \boldsymbol{a}+\frac{q}{c} \boldsymbol{A}\right)
$$

Taking curl of (31) leads to

$$
\left(\square+\frac{1}{\lambda^{2}}\right) \boldsymbol{B}=\frac{\hbar c}{\lambda^{2} q} \boldsymbol{\omega},
$$

where the penetration depth $\lambda$ is defined as

$$
\lambda=\sqrt{m c^{2} / \rho_{0} q^{2}} \quad .
$$

Eq. (32) represents the Meissner effect that the magnetic field has a penetration depth. Next let us integrate eq. (32) in the region $D$ surrounded by a closed path.

$$
\int_{D}\left(\lambda^{2} \square+1\right) \boldsymbol{B} \cdot d \boldsymbol{n}=\frac{c \hbar}{q} \int_{D} \boldsymbol{\omega} \cdot d \boldsymbol{n}=\frac{c \hbar}{q} 2 \pi n \equiv \phi_{0} n .
$$

If we choose the integration region $D$ larger than the penetration depth around the vortex, $\square \boldsymbol{B}$ can be neglected because there are neither a magnetic field nor an electric current. Therefore the whole magnetic flux coincides with the integer multiple of unit magnetic flux $\phi_{0}=(c \hbar / q) 2 \pi$.

The Hall (or transverse) voltage and the longitudinal voltage are produced depending on the direction of the vortex motion, which follow from the Maxwell's equations: If a magnetic flux moves, the magnetic field changes in time only through the flux motion and an electric field is produced by

$$
\begin{aligned}
\nabla \times \boldsymbol{E}(x)=-\frac{1}{c} \dot{\boldsymbol{B}}(x) & =\frac{1}{c}(\dot{\boldsymbol{X}} \cdot \nabla) \boldsymbol{B}(x)=-\frac{1}{c} \nabla \times(\dot{\boldsymbol{X}} \times \boldsymbol{B}(x)) \\
\rightarrow \boldsymbol{E} & =-\frac{1}{c} \dot{\boldsymbol{X}} \times \boldsymbol{B}
\end{aligned}
$$


Now let us examine vortex dynamics in a superconductor by taking the variation of our effective action (29) of the "classical" level. Since $\boldsymbol{X}$ dependence arises only through the source term as with the superfluid vortex system, the obtained force acting on vortices in a superconductor is also the Magnus force (77). The Magnus force (7) is rewritten in terms of the magnetic field through eq.(32)

$$
-m \rho_{0}(\dot{\boldsymbol{X}}-\boldsymbol{v}) \times \boldsymbol{\omega}=\frac{q}{c} \rho_{0}(\boldsymbol{v}-\dot{\boldsymbol{X}}) \times\left(1+\lambda^{2} \square\right) \boldsymbol{B},
$$

where the local fluid velocity is related to the electric current by $\boldsymbol{v}=\left(c / q \rho_{0}\right) \boldsymbol{J}_{e}$. When the vortex is almost at rest $(\dot{\boldsymbol{X}} \simeq 0)$ and the photon momentum is smaller than $1 / \lambda\left(\lambda^{2} \square \boldsymbol{B} \simeq 0\right)$, the right hand side of (36) reduces to the Lorentz force, $(1 / c) \boldsymbol{J}_{e} \times \boldsymbol{B}[\mathbf{1}]$.

Now let us evaluate the effective action for vortices in charged superfluids by taking into account the phonon and photon fluctuations. Analogous to the neutral superfluid, a stationary solution without vortex singularities reads

$$
\begin{array}{r}
a_{\mu}=0 \quad, \quad \rho=\rho_{0} \quad, \quad \frac{\hbar}{m} \nabla \theta_{b g}-\frac{q}{m c} \boldsymbol{A}_{b g}=\boldsymbol{v}_{b g} \quad \text { and } \quad \dot{\theta}_{b g}=-\frac{m}{2 \hbar} \boldsymbol{v}_{b g}^{2} \\
\partial^{\mu} F_{\mu 0, b g}=\frac{q}{c} \rho_{0}+\rho_{l a t}=0 \quad, \quad \partial^{\mu} F_{\mu i, b g}=-\frac{q}{c} \rho_{0} v_{b g}^{i} .
\end{array}
$$

Deep inside bulk superconductors in three dimensions, neither the magnetic field nor the electric current present. On the other hand, in the surface region of a bulk superconductor and in a thin superconducting film, the electric current is non-vanishing due to the effect of the boundary conditions. These features are a consequence of the above equations. In the following, we will mostly consider a thin film placed in the $x-y$ plane and assume that the transport current (the background velocity) is constant in the $x-y$ direction.

By expanding $\rho(x), \theta(x)$ and $A_{\mu}(x)$ in (29) around the above solution (37), we get an effective action

$$
\mathcal{Z}=\int \mathcal{D}\left[\delta \rho, \delta \theta, \delta A_{\mu}, a_{\mu}, b_{\mu \nu}\right] e^{i S\left(\rho, \theta, A_{\mu}, a_{\mu}, b_{\mu \nu} ; X\right)}=e^{i S_{e f f}(X)}
$$

where 


$$
\begin{aligned}
\mathcal{L}= & \mathcal{L}_{s t}+\delta \rho\left(\hbar a_{0}-\hbar \boldsymbol{v}_{b g} \cdot \boldsymbol{a}-\frac{\hbar^{2}}{2 m} \boldsymbol{a}^{2}\right)+\frac{q \rho_{0}}{c} \delta \boldsymbol{A} \cdot \frac{\hbar}{m} \boldsymbol{a} \\
& +\frac{1}{2}\left(\begin{array}{llll}
\delta \rho & \delta \theta & \delta A_{0} & \delta \boldsymbol{A}
\end{array}\right) G^{-1}\left(\begin{array}{c}
\delta \rho \\
\delta \theta \\
\delta A_{0} \\
\delta \boldsymbol{A}
\end{array}\right)-\delta \rho \hbar \boldsymbol{a} \cdot \frac{\hbar}{m}\left(\nabla \delta \theta-\frac{q}{c \hbar} \delta \boldsymbol{A}\right)+O\left(\delta^{3}\right)+(b f, b J), \\
\mathcal{L}_{s t}= & \rho_{0}\left(\hbar a_{0}-\hbar \boldsymbol{v}_{b g} \cdot \boldsymbol{a}-\frac{\hbar^{2}}{2 m} \boldsymbol{a}^{2}\right)-\frac{1}{4} F_{b g, \mu \nu} F_{b g}^{\mu \nu},
\end{aligned}
$$

with Hermitian matrix $G^{-1}$

$$
G^{-1}=\left(\begin{array}{cccc}
\left(-2 g+\frac{\hbar^{2}}{4 m \rho_{0}} \triangle\right) & -\hbar\left(\partial_{0}+\boldsymbol{v}_{b g} \cdot \nabla\right) & -q / c & \frac{q}{c} \boldsymbol{v}_{b g} \\
\hbar\left(\partial_{0}+\boldsymbol{v}_{b g} \cdot \nabla\right) & \frac{\rho_{0} \hbar^{2}}{m} \Delta & 0 & 0 \\
-q / c & 0 & -\triangle & 0 \\
\frac{q}{c} \boldsymbol{v}_{b g} & 0 & 0 & -\left(\square+\frac{1}{\lambda^{2}}\right)
\end{array}\right) .
$$

Here the Coulomb gauge for the $a$-field and $\delta A$-field are used. For zero background velocity, $G$ has poles at

$$
\omega^{2}=\frac{1}{\lambda^{2}}+\boldsymbol{k}^{2}, \quad \omega^{2}=\frac{1}{\lambda^{2}}+\epsilon^{2}\left(\boldsymbol{k}^{2}\right) \quad,
$$

where $\epsilon$ is the Bogoliubov spectrum (14). The massless Goldstone mode has been absorbed into massive mode. In order to take into account density fluctuations and the photon effect, we integrate out these fluctuations;

$$
\begin{aligned}
\mathcal{L}_{e f f}\left(a_{\mu}, b_{\mu \nu} ; X\right) \approx \mathcal{L}_{s t} \\
\quad-\frac{\rho_{0}}{2 m}\left(\hbar\left(a_{0}-\boldsymbol{v}_{b g} \cdot \boldsymbol{a}\right)-\frac{\hbar^{2}}{2 m} \boldsymbol{a}^{2}\right) \frac{\triangle}{\left(\partial_{0}+\boldsymbol{v}_{b g} \cdot \nabla\right)^{2}+\epsilon^{2}+1 / \lambda^{2}}\left(\hbar\left(a_{0}-\boldsymbol{v}_{b g} \cdot \boldsymbol{a}\right)-\frac{\hbar^{2}}{2 m} \boldsymbol{a}^{2}\right) \\
\quad+\frac{\rho_{0} \hbar^{2}}{2 m} \boldsymbol{a} \cdot \frac{1 / \lambda^{2}}{\square+1 / \lambda^{2}} \boldsymbol{a}+O\left(\boldsymbol{v}^{2} / c_{s}^{2}\right) .
\end{aligned}
$$


Suppose that a thin superconducting sample is set in the $x-y$ plane and the magnetic field is applied in the $z$-direction. Vortices lie along the $z$ direction. Suppose also that the transport current $\boldsymbol{J}_{e}$ flows in the $x-y$ plane. By carrying out the integration of the $b$-field and using the solutions (19), the first term of the effective action (42) becomes

$$
\begin{aligned}
-\frac{\rho_{0} \hbar^{2}}{2 m} \int & d^{2} x \quad\left(a_{0}-\boldsymbol{v}_{b g} \cdot \boldsymbol{a}\right)\left(\frac{\triangle}{\left(\partial_{0}+\boldsymbol{v}_{b g} \cdot \nabla\right)^{2}+\epsilon^{2}+1 / \lambda^{2}}\right)\left(a_{0}-\boldsymbol{v}_{b g} \cdot \boldsymbol{a}\right) \\
\approx & \frac{1}{8 \pi^{2}}\left(\frac{\phi_{0}}{\lambda c_{s}}\right)^{2}\left(\sum_{a=b}+\sum_{a \neq b}\right) n_{a} n_{b}\left(\dot{\boldsymbol{X}}_{a}-\boldsymbol{v}_{b g}\right)^{i}\left(\dot{\boldsymbol{X}}_{b}-\boldsymbol{v}_{b g}\right)^{j} \epsilon^{i k} \epsilon^{j l} \\
& \frac{\triangle_{X}}{\triangle_{X}-1 /\left(\lambda c_{s}\right)^{2}} \int d^{2} x \partial_{k} \ln \left|\boldsymbol{x}-\boldsymbol{X}_{a}\right| \partial_{l} \ln \left|\boldsymbol{x}-\boldsymbol{X}_{b}\right| \\
= & \sum_{a} \frac{1}{2} m_{e f f, a}\left(\dot{\boldsymbol{X}}_{a}-\boldsymbol{v}_{b g}\right)^{2} \\
& +\frac{1}{8 \pi}\left(\frac{\phi_{0}}{\lambda c_{s}}\right)^{2} \sum_{a \neq b} n_{a} n_{b}\left\{\left(\dot{\boldsymbol{X}}_{a}-\boldsymbol{v}_{b g}\right) \cdot\left(\dot{\boldsymbol{X}}_{b}-\boldsymbol{v}_{b g}\right)\left(K_{0}\left(\frac{\left|\boldsymbol{X}_{a b}\right| c}{\lambda c_{s}}\right)+\frac{1}{\triangle-1 /\left(\lambda c_{s}\right)^{2}} \frac{2}{\left|X_{a b}\right|^{2}}\right)\right. \\
& \left.-\left(\dot{\boldsymbol{X}}_{a}-\boldsymbol{v}_{b g}\right) \cdot\left(\frac{4}{\triangle-1 /\left(\lambda c_{s}\right)^{2}} \frac{\boldsymbol{X}_{a b} \boldsymbol{X}_{a b}}{\boldsymbol{X}_{a b}^{4}}\right) \cdot\left(\dot{\boldsymbol{X}}_{b}-\boldsymbol{v}_{b g}\right)\right\}
\end{aligned}
$$

Here $2 \pi K_{0}(x)=i \pi^{2} H_{0}(i x)$ with $H_{0}$ being a Hankel function of the first kind. The time derivative $\left(\partial_{0}-\boldsymbol{v} \cdot \nabla\right)^{2}$ is neglected because $\dot{\boldsymbol{X}}$ and $\boldsymbol{v}$ are much smaller than $c_{s}$, and the Bogoliubov spectrum $\epsilon^{2}$ is approximated by $-c_{s}^{2} \triangle$ in the adiabatic approximation. In the last line of (43) the interaction part is neglected, because $\left|\boldsymbol{X}_{a b}\right| c>>\lambda c_{s}$.

One should note the qualitative difference between the kinetic term in the superfluid case (27) and that in the superconductor case (43). "Center of vorticity" enters into the kinietic term in the former, while each vortex has separate contributions to the kinetic term in the latter. In fact, in the superconductor, charge screening makes a vortex-size finite and each vortex can carry individual mobility and energy. The inertial mass of a single vortex is estimated using the previous regularization with the coherence length $\xi$ as an ultraviolet cut off,

$$
m_{e f f, a}=\frac{1}{4 \pi}\left(\frac{\phi_{0}}{\lambda c_{s}}\right)^{2} n_{a}^{2} K_{0}\left(\frac{\xi c}{\lambda c_{s}}\right)
$$




$$
\approx \frac{1}{4 \pi}\left(\frac{\phi_{0}}{\lambda c_{s}}\right)^{2} n_{a}^{2} \begin{cases}\ln \frac{\lambda c_{s}}{\xi c} & , \quad \xi c<<\lambda c_{s} \\ \sqrt{\frac{\lambda \pi c_{s}}{2 \xi c}} e^{-\xi c / \lambda c_{s}} & , \quad \xi c>>\lambda c_{s}\end{cases}
$$

So far, we have been adopting the vorticity distribution with the $\delta$-function form (30), for simplicity. In this approximation, the ultraviolet cutoff should be the coherence length $\xi$. For neutral superfluids, $\xi$ is nearly equal to the atomic scale $d$ which is the cutoff of the theory, so the $\delta$-function vorticity is a good approximation. For superconductors, however, $\xi$ is larger than the atomic scale, so the vortex-core contribution could be non-negligible. In order to estimate the core contribution, let us separate the integral of the left hand side of (43) as $\int_{r=\xi}^{\infty} d^{2} x \rightarrow\left(\int_{r=d}^{\xi}+\int_{r=\xi}^{\infty}\right) d^{2} x$ : The second term, which is a contribution from outside the core, is given by (43) and (44). The first term from inside the core, can be evaluated in the following way. In the vorticity tensor, the $\delta$-function is replaced by the regularized function, for example

$$
\delta^{(4)}(x-X) \rightarrow \delta\left(x_{0}-X_{0}\right) \delta\left(x_{3}-X_{3}\right)\left(\frac{1}{\sqrt{\pi} \xi}\right)^{2} e^{-\left(\frac{\boldsymbol{x}_{-} \boldsymbol{X}}{\xi}\right)^{2}}
$$

which is introduced in [13]. So the vorticity vector $\boldsymbol{\omega}$ has a finite peak at $X$ with width $\xi$. By solving the constraint (17), one can show that the velocity around a vortex, $\boldsymbol{a}$, has a maximum value at the edge of a core and decreases toward a vortex center, then vanishes at the vortex center. So we replace $\nabla \ln r$ in $a_{\mu}$ by $\left(\boldsymbol{r} / \pi \xi^{2}\right) P(r / \xi)$ where $P$ is a solution of (17); $\nabla \cdot\left(\boldsymbol{r} P(r / \xi) / \pi \xi^{2}\right)=e^{-((\boldsymbol{x}-\boldsymbol{X}) / \xi)^{2}} /\left(\pi \xi^{2}\right)$. One can also show that $a_{\mu}$ has linear dependence in $\boldsymbol{r}$ near the vortex center with $P(0)=1$. Then the core contribution becomes

$$
\begin{gathered}
\frac{1}{2}\left(\frac{\phi_{0}}{\lambda c_{s}}\right)^{2} n_{a}^{2} \int_{r=d}^{\xi} d^{2} x \quad \dot{\boldsymbol{X}}^{2} \frac{\boldsymbol{r} P(r / \xi)}{\pi \xi^{2}} \cdot\left(\frac{\triangle}{\triangle-1 /\left(\lambda c_{s}\right)^{2}}\right) \frac{\boldsymbol{r} P(r / \xi)}{\pi \xi^{2}} \\
=\frac{1}{2}\left(\frac{\phi_{0}}{\lambda c_{s}}\right)^{2} n_{a}^{2} \alpha\left(\frac{d}{\xi}, \frac{1}{\lambda c_{s}}\right) \dot{\boldsymbol{X}}^{2}
\end{gathered}
$$

where

$$
\alpha(\Lambda, \mu) \equiv \frac{1}{\pi^{2}} \int_{\Lambda}^{1} d^{2} \hat{r} \quad \hat{\boldsymbol{r}} P(\hat{r}) \cdot\left(\frac{\hat{\triangle}}{\hat{\triangle}-\mu^{2}}\right) \hat{\boldsymbol{r}} P(\hat{r}) \quad,
$$


with $\hat{\boldsymbol{r}} \equiv \boldsymbol{r} / \xi$. For a small core, $\xi \sim d, \alpha$ is almost zero, since $\alpha(\Lambda, \mu) \approx \frac{2}{\pi}(1-\Lambda)[\hat{r} \hat{\boldsymbol{r}} P(\hat{r})$. $\left.\left(\frac{\hat{\Delta}}{\hat{\Delta}-\mu^{2}}\right) \hat{\boldsymbol{r}} P(\hat{r})\right]\left.\right|_{\hat{r}=(1-\Lambda) / 2}$. For a large core, $\xi>>d, \alpha$ becomes a constant of order 1 , since $\left(\frac{\hat{\triangle}}{\hat{\triangle}-\left(\xi c / \lambda c_{s}\right)^{2}}\right)$ takes 1 for $\hat{\triangle}>>\left(\xi c / \lambda c_{s}\right)^{2}$, constant of order 1 for $\hat{\triangle} \approx\left(\xi c / \lambda c_{s}\right)^{2}$, and 0 for $\hat{\triangle}<<\left(\xi c / \lambda c_{s}\right)^{2}$. The resultant effective mass including with the contribution from outside the core is

$$
m_{e f f, a}^{t o t}=\frac{1}{4 \pi}\left(\frac{\phi_{0}}{\lambda c_{s}}\right)^{2} n_{a}^{2}\left(K_{0}\left(\frac{\xi c}{\lambda c_{s}}\right)+\alpha\left(\frac{d}{\xi}, \frac{1}{\lambda c_{s}}\right)\right)
$$

Our result is consistent with that in ref. [18 for $\xi c<<\lambda c_{s}$. However, our formula is not limited to this parameter region, while that of [18] is only valid in this region. For conventional superconductors, we have the Landau parameter $\lambda / \xi \approx 10$ and a sound velocity $c_{s} \approx 10^{-4} c$, while for high $T_{c}$ superconductors we have $\lambda / \xi \approx 10^{3}$ and $c_{s} \approx 10^{-3} c$. Thus the core of vortices in conventional superconductors is relatively large, $d / \xi \sim 10^{-4}, \alpha \sim 10^{-6}$ and $K_{0} \sim 10^{-400}$. On the other hand, that in high $T_{c}$ superconductors is small, $d / \xi \sim 10^{0 \sim-1}$, $\alpha \sim 0$ and $K_{0} \sim 0.4$. Therefore, the effective vortex mass in a conventional superconductor mainly comes from inside a core, while that of high $T_{c}$ superconductor mainly comes from outside the core.

Now let us return to the evaluation of the $\boldsymbol{a}^{2}$ term in $S_{\text {eff }}\left(a_{\mu} ; X\right)$. The second term of the effective action (42), when added to the term in the stationary action, $\mathcal{L}_{s t}$, becomes

$$
\begin{aligned}
-\frac{\rho_{0} \hbar^{2}}{2 m} \int & d^{2} x \boldsymbol{a} \cdot\left(1-\frac{1 / \lambda^{2}}{\square+1 / \lambda^{2}}\right) \boldsymbol{a} \\
& \approx-\frac{1}{8 \pi^{2}}\left(\frac{\phi_{0}}{\lambda}\right)^{2}\left(\sum_{a=b}+\sum_{a \neq b}\right) n_{a} n_{b} \frac{\triangle_{X}}{\triangle_{X}-1 / \lambda^{2}} \int d^{2} x \partial_{i} \ln \left|\boldsymbol{x}-\boldsymbol{X}_{a}\right| \partial_{i} \ln \left|\boldsymbol{x}-\boldsymbol{X}_{b}\right| \\
& =-\sum_{a} E_{0, a}-\frac{1}{4 \pi}\left(\frac{\phi_{0}}{\lambda}\right)^{2} \sum_{a \neq b} n_{a} n_{b} K_{0}\left(\frac{\left|\boldsymbol{X}_{a b}\right|}{\lambda}\right) \\
& \approx-\sum_{a} E_{0, a}-\frac{1}{4 \pi}\left(\frac{\phi_{0}}{\lambda}\right)^{2} \sum_{a \neq b} n_{a} n_{b} \begin{cases}\ln \frac{\lambda}{\left|\boldsymbol{X}_{a b}\right|} & ,\left|\boldsymbol{X}_{a b}\right|<<\lambda \\
\sqrt{\frac{2 \pi \lambda}{\left|\boldsymbol{X}_{a b}\right|}} e^{-\left|\boldsymbol{X}_{a b}\right| / \lambda},\left|\boldsymbol{X}_{a b}\right|>>\lambda\end{cases}
\end{aligned}
$$

where the vortex energy is estimated to be 


$$
\begin{aligned}
E_{0, a} & =\frac{1}{4 \pi}\left(\frac{\phi_{0}}{\lambda}\right)^{2} n_{a}^{2} K_{0}\left(\frac{\xi}{\lambda}\right) \\
& \approx \frac{1}{4 \pi}\left(\frac{\phi_{0}}{\lambda}\right)^{2} n_{a}^{2} \ln \frac{\lambda}{\xi}, \quad \xi<<\lambda .
\end{aligned}
$$

The total vortex energy with the core contribution reads

$$
\begin{aligned}
E_{0, a}^{t o t} & =\frac{1}{4 \pi}\left(\frac{\phi_{0}}{\lambda}\right)^{2} n_{a}^{2}\left(K_{0}\left(\frac{\xi}{\lambda}\right)+\alpha\left(\frac{d}{\xi}, \frac{1}{\lambda}\right)\right) \\
& \approx \frac{1}{4 \pi}\left(\frac{\phi_{0}}{\lambda}\right)^{2} n_{a}^{2} \ln \frac{\lambda}{\xi} \quad, \quad \xi<<\lambda .
\end{aligned}
$$

The first part of the resultant $E_{0}^{\text {tot }}$ coincides with the free energy of a vortex line per unit length given by Abrikosov [22]. The core contribution is generally small; for the conventional superconductor case $K_{0} \sim 3$ and $\alpha \sim 0.1$ and for the high $T_{c}$ superconductor case $K_{0} \sim 1$ and $\alpha \sim 0$

The vortex-vortex interaction in (49) has a natural form: At small distances, the same logarithmic force as the superfluid vortices acts between the vortices. For large distances, the force is exponentially suppressed because of the electromagnetic shielding in superconductors. Other vortex interactions are suppressed by the factor $\boldsymbol{v}^{2} / c_{s}^{2}$.

The resultant effective action for vortices in a charged superfluid up to $O\left(\hbar^{2}\right)$ and $O\left(\left(1 / c_{s}\right)^{0}\right)$ is given by

$$
S_{e f f}(X)=\int d t\left[\int d^{2} x \mathcal{L}_{e m}+\sum_{a}\left\{L_{B e r r y, a}+L_{v e, a}+L_{k i n, a}\right\}+L_{i n t}\right]-U_{\text {pin }}
$$




$$
\left\{\begin{array}{l}
\mathcal{L}_{e m}=\frac{1}{2}\left(\boldsymbol{E}_{b g}^{2}-\boldsymbol{B}_{b g}^{2}\right) \\
L_{\text {Berry,a }}=\rho_{0} \pi \hbar n_{a} \boldsymbol{e}_{z} \cdot\left(\dot{\boldsymbol{X}}_{a}-\boldsymbol{v}_{b g}\right) \times\left(\boldsymbol{X}_{a}-\boldsymbol{v}_{b g} t\right) \\
L_{v e, a}=-E_{0, a}^{t o t}, \quad \text { with } \quad E_{0, a}^{t o t}=\frac{1}{4 \pi}\left(\phi_{0} / \lambda\right)^{2} n_{a}^{2}\left(K_{0}\left(\frac{\xi}{\lambda}\right)+\alpha\left(\frac{d}{\xi}, \frac{1}{\lambda}\right)\right) \\
L_{k i n, a}=\frac{1}{2} m_{e f f, a}^{t o t}\left(\dot{\boldsymbol{X}}_{a}-\boldsymbol{v}_{b g}\right)^{2}, \quad \text { with } m_{e f f, a}^{t o t}=\frac{1}{4 \pi}\left(\phi_{0} / \lambda c_{s}\right)^{2} n_{a}^{2}\left(K_{0}\left(\frac{\xi c}{\lambda c_{s}}\right)+\alpha\left(\frac{d}{\xi}, \frac{1}{\lambda c_{s}}\right)\right) \\
L_{i n t}=-\frac{1}{4 \pi}\left(\frac{\phi_{0}}{\lambda}\right)^{2} \sum_{a \neq b} n_{a} n_{b} K_{0}\left(\frac{\left|\boldsymbol{X}_{a b}\right|}{\lambda}\right)
\end{array}\right.
$$

where $\mathcal{L}_{e m}$ is the electromagnetic Lagrangian.

The equation of motion for an isolated vortex is determined by varying the effective action which is the sum of the acceleration term, the Magnus force and the pinning force. A scenario for vortex motion is as follows: At first, a vortex in pinned, so that $\dot{\boldsymbol{X}}$ is almost zero. If the transport current is large enough, the Magnus force becomes strong so that the vortex starts to move by overcoming the pinning potential. A longitudinal voltage is thus produced. The Magnus force acts in such a way that the vortex velocity gets closer to the transport velocity. Eventually the vortex moves along with the transport current. As a result, Hall (transverse) voltage is produced.

\section{SUMMARY AND CONCLUDING REMARKS}

We have presented a unified treatment of vortex dynamics at zero temperature by using the topological Landau-Ginzburg theory. It is shown that the rank-two antisymmetric tensor potential, $b_{\mu \nu}$-field, is a fundamental field coupled to vortex lines, such that the local coupling " $b_{\mu \nu} J^{\mu \nu " ~ a n d ~ t h e ~ t o p o l o g i c a l ~ t e r m ~ " ~} \epsilon^{\mu \nu \rho \lambda} b_{\mu \nu} f_{\rho \lambda}$ " give rise to the Magnus force in both neutral and charged superfluids. Analogies with the electromagnetic theory have been shown; the rank-two antisymmetric tensor potential, the hydrodynamical current and the 
Magnus force correspond to the vector potential, the electromagnetic field and the Lorentz force, respectively.

Although we treat $b_{\mu \nu}$ as a Lagrange multiplier in this paper, the action written in terms of $b_{\mu \nu}$ after integrating out the $a_{\mu}$ field in our theory (1) will have the same structure as the Kalb-Ramond type theory [24]. In this case, the $b_{\mu \nu}$-field becomes dynamical. This may clarify the relation between our theory and that in [12,23] where $b_{\mu \nu}$ is treated as a dynamical field with a $H_{\mu \nu \lambda}^{2}$ term.

The phonon effect in a neutral superfluid was calculated and our main result is the effective action given by (27). In our derivation, " $\rho_{0} \hbar\left(a_{0}-\boldsymbol{v}_{b g} \cdot \boldsymbol{a}\right)$ " becomes the Berry phase term after integrating out the $b_{\mu \nu}$-field and it leads naturally to the Magnus force. In deriving the Berry phase term and the Magnus force, a first order formalism with single time-derivative is essential: Phenomenological Landau-Ginzburg theory having second order time-derivative (such as that in [18]) cannot lead to the Berry phase term.

We provide a systematic method to regulate vortex self-interactions such as the vortex energy and the inertial mass. It turned out that the vortex energy and the inertial mass in neutral superfluids can only be defined for the whole system and not for the individual vortices. In fact, the vortex energy is proportional to the square of the total vorticity. Also, the real dynamical degree of freedom is "the center of vorticity" of the system as is seen from the effective kinetic term.

The obtained energy $E_{0}$ for an isolated vortex coincides with Feynman's chemical potential. The obtained inertial mass is about $m \rho_{0} \gamma_{0}^{2} / c_{s}^{2}\left(m\right.$ is the atomic mass, $\rho_{0}$ is the condensation density, $\gamma_{0}$ is the unit vorticity and $c_{s}$ is the zero sound velocity). They satisfy the relation; $m_{e f f}=E_{0} / c_{s}^{2}$ which is consistent with the result in [18] where phenomenological Landau-Ginzburg theory is used supplemented with the Fermi-liquid theory. We have clarified the origin of the inertial mass by dividing fields into the stational configuration and the zero sound wave: a vortex follows the classical hydrodynamical law, " $\dot{X}=\boldsymbol{v}$ ", if the 
zero sound wave is neglected. Once the zero sound wave is taken into account, a vortex gets an inertial mass and deviation from the classical hydrodynamics arises. Therefore the zero sound wave should play an essential role for the pinning and depinning phenomena and also for the quantum tunneling of vortices.

An effective action with photon and density fluctuations in charged superfluids is given by (52). The fundamental force acting on vortices in superconductors is also the Magnus force. For a slowly moving magnetic flux (vortex), the magnus force reduces to " $\boldsymbol{J}_{e} \times \boldsymbol{B}$ " which is the origin of the Lorentz force between the magnetic flux and the transport current. Vortices get inertial mass through $\delta A_{0}$ and $\delta \rho$ fluctuations. In contrast to the superfluid case, the vortex energy and the inertial mass can be defined for individual vortices because of the charge screening in superconductors. Core contributions to the vortex energy and the

inertial mass are also evaluated. The obtained inertial mass is about $10^{-6}(1 / 4 \pi)\left(\phi_{0} / \lambda c_{s}\right)^{2}$ for conventional superconductor and $0.4(1 / 4 \pi)\left(\phi_{0} / \lambda c_{s}\right)^{2}$ for high $T_{c}$ superconductor, where $\phi_{0}$ is the unit magnetic flux and $\lambda$ is the penetration depth. Thus the main contribution to the effective inertial mass for conventional superconductors comes from inside the core, while that for high $T_{c}$ superconductor comes from outside the core. In reference [18] opposite results are obtained for high $T_{c}$ superconductors. The reason for this discrepancy comes from the unjustified extrapolation of the formula in [18] from the region $\xi c<<\lambda c_{s}$ to the region $\xi c \approx \lambda c_{s}$. We do not have such a problem, since our formula is valid for all parameter space.

Finally, we mention that the inertial mass of a vortex may be determined experimentally by the real-time observation of the vortex motion using electron microscopy 25.

\section{ACKNOWLEDGMENTS}

We wish to thank P.Ao for fruitful discussions, critical comments and collaboration in early stage of this work. We are grateful to D.J.Thouless for instructive discussions. We also wish to thank B.Hanlon for reading manuscript. S.Y. was supported by Grant-in- 
Aid for Scientific Reserch from Ministry of Education, Science and Culture (grant number 07854012). M.S. is Fellow of the Japan Society for the Promotion of Science for Japanese Junior Scientists. T. H. was supported by Grant-in-Aid for Scientific Reserch from Ministry of Education, Science and Culture (grant number 06102004). 


\section{APPENDIX: A}

In this appendix we derive the integral formula (21):

$$
\begin{aligned}
& \int d^{2} x \partial_{i} \ln \left|\boldsymbol{x}-\boldsymbol{X}_{a}\right| \partial_{j} \ln \left|\boldsymbol{x}-\boldsymbol{X}_{b}\right| \\
& \quad= \begin{cases}-\pi\left[\delta^{i j}\left\{\ln \left(\left|\boldsymbol{X}_{a b}\right| / \varepsilon e^{1 / 2}\right)-\ln \left(R / \varepsilon e^{1 / 2}\right)\right\}+\left(X_{a b}^{i} X_{a b}^{j} /\left|\boldsymbol{X}_{a b}\right|^{2}-\delta^{i j} / 2\right)\right], & \text { for } \boldsymbol{X}_{a} \neq \boldsymbol{X}_{b} \\
\pi \delta^{i j} \ln \left(R / \varepsilon e^{1 / 2}\right) & , \text { for } \boldsymbol{X}_{a}=\boldsymbol{X}_{b}\end{cases}
\end{aligned}
$$

In the left hand side of (A1) there is an ultraviolet divergence at $\boldsymbol{X}_{a}=\boldsymbol{X}_{b}$, so we regulate $\ln |x|$ as $\ln \sqrt{x^{2}+\varepsilon^{2}}$ with a small parameter $\varepsilon$. For an infrared divergence, we introduce a large cutoff parameter $R$ so that we neglect $1 / R^{2}$ terms. Using Feynman's parameter formula

$$
\frac{1}{a b}=\int_{0}^{1} d t \frac{1}{[a t+b(1-t)]^{2}}
$$

the left hand side of (A1) becomes

$$
\begin{aligned}
\int d^{2} x & \frac{\left(x^{i}-X_{a}^{i}\right)\left(x^{j}-X_{b}^{j}\right)}{\left(\left|\boldsymbol{x}-\boldsymbol{X}_{a}\right|^{2}+\varepsilon^{2}\right)\left(\left|\boldsymbol{x}-\boldsymbol{X}_{b}\right|^{2}+\varepsilon^{2}\right)} \\
= & \int_{0}^{1} d t \int d^{2} x^{\prime} \frac{\left(x^{\prime i}+X_{a b}^{i}(t-1)\right)\left(x^{\prime j}+X_{a b}^{j} t\right)}{\left[\boldsymbol{x}^{\prime 2}+t(1-t) \boldsymbol{X}_{a b}^{2}+\varepsilon^{2}\right]^{2}} \\
= & -\pi\left[\delta^{i j}\left\{-\frac{1}{2}+\ln \frac{\varepsilon}{R}-\sqrt{\frac{1}{4}+\frac{\varepsilon^{2}}{\left|\boldsymbol{X}_{a b}\right|^{2}}} \ln \mid \frac{1 / 2-\sqrt{1 / 4+\varepsilon^{2} /\left|\boldsymbol{X}_{a b}\right|^{2}}}{1 / 2+\sqrt{1 / 4+\varepsilon^{2} /\left|\boldsymbol{X}_{a b}\right|^{2}} \mid}\right\}\right. \\
& \left.+\frac{X_{a b}^{i} X_{a b}^{j}}{\left|\boldsymbol{X}_{a b}\right|^{2}}\left(1+\frac{\varepsilon^{2}}{\left|\boldsymbol{X}_{a b}\right|^{2}} \frac{1}{\sqrt{1 / 4+\varepsilon^{2} /\left|\boldsymbol{X}_{a b}\right|^{2}}} \ln \left|\frac{1 / 2-\sqrt{1 / 4+\varepsilon^{2} /\left|\boldsymbol{X}_{a b}\right|^{2}}}{1 / 2+\sqrt{1 / 4+\varepsilon^{2} /\left|\boldsymbol{X}_{a b}\right|^{2}}}\right|\right)\right]
\end{aligned}
$$

It is easy to recover the right hand side of (A1) for both $\boldsymbol{X}_{a} \neq \boldsymbol{X}_{b}$ with $\left|\boldsymbol{X}_{a b}\right|>>\varepsilon$ and $\boldsymbol{X}_{a}=\boldsymbol{X}_{b}$. Also, the above formula (A3) may be useful for a case where two vortices are close to each other $\left|\boldsymbol{X}_{a b}\right| \sim \varepsilon$. 


\section{REFERENCES}

[1] See for example, Y.B.Kim and M.J.Stephen, Superconductivity edited. by R.D.Parks, (Dekker,New York,1969), F.G.de Gennes, Superconductivity of metals and alloys, (W.A.Benjamin, inc. 1965).

[2] D.R.Tilley and J.Tilley, Superfuidity and Superconductivity ,(Adam Hilger, Bristol, 1990).

[3] V.T.Ginzburg and P.Pitaevskii, Sov. Phys. JETP 7 (1958) 858, P.Pitaevskii, Sov. Phys. JETP 13 (1961) 451, E.P.Gross Nuovo Cimento 20 (1961) 454.

[4] T.W.Jing and N.P.Ong, Phys. Rev. B42 (1990) 10781, and references cited therein.

[5] S.J.Hagen et al., Phys. Rev. B47 (1993) 1064, and references cited therein.

[6] P.Ao and D.J.Thouless, Phys. Rev. Lett. 70 (1993) 2158.

[7] P.Ao and D.J.Thouless, Phys. Rev. Lett. 72 (1994) 132.

[8] Q.Niu, P.Ao and D.J.Thouless, Phys. Rev. Lett. 72 (1994) 1706.

[9] M.Hatsuda, S.Yahikozawa, P.Ao and D.J.Thouless, Phys. Rev. B49 (1994) 15870.

[10] F.Gaitan, Phys. Rev. B51 (1995) 9061, J. Phys. Cond. Mat. 7 (1995) L165.

[11] K.Lee, cond-mat/9409046, "Vortices and sound waves in superfluids".

[12] A. Zee, Nucl. Phys. B421 (1994) 111.

[13] M.Sato and S.Yahikozawa, Nucl. Phys. B436 (1995) 100.

[14] H.Kuratsuji and H.Yabu, TMU-NT 940802, "Magnus force for a single superfluid vortex: Hamiltonian dynamics approach based on the Ginzburg-Landau Lagrangian".

[15] H.Lamb, "Hydrodynamics", (Cambridge University press, 6ed. 1932) §146 and $§ 69$.

[16] A.T.Dorsey, Phys. Rev. B46 (1992) 8376. 
[17] A.O.Calderia and A.J.Leggett, Annals of Phys. 149 (1983) 374,

G.Blatter, V.B.Geshkenbein and V.M.Vinokur, Phys. Rev. Lett. 66 (1991) 3297,

G.Blatter and V.B.Geshkenbein, Phys. Rev. B47 (1993) 2725,

M.J.Stephen, Phys. Rev. Lett. 72 (1994) 1534.

[18] J-M. Duan and A.J.Leggett, Phys. Rev. Lett. 68 (1992) 1216,

J-M. Duan, Phys. Rev. B48 (1993) 333.

[19] V.N.Popov, Theor. Mat. Fiz., 6 (1971) 90 (65,English version),

Theor. Mat. Fiz., 11 (1972) 236 (478,English), Theor. Mat. Fiz., 11 (1972) 354 (565,English); Functional interals and collective excitaions, (Cambridge university press, 1987).

[20] F.D.M.Haldane and Y.S.Wu, Phys. Rev. Lett. 55 (1985) 2887.

[21] R.P.Feynman, chapter II in vol. I, Progress in Low Temperature Physics, edited by C.J.Gorter (1955).

[22] Abrikosov, Sov. Phys. JETP 5 (1957) 1174.

[23] R.L.Davis, Physica B178 (1992) 76.

[24] M.Kalb and P.Ramond, Phys.Rev. D9 (1974) 2273.

[25] K.Harada et.al., Nature 360 (1992) 51. 